Family Medicine and Community Health

\section{Using electronic health record's data to assess daily dose of opioids prescribed for outpatients with chronic non- cancer pain}

\author{
Wen-Jan Tuan (D) , ${ }^{1}$ Nalini Sehgal, ${ }^{2}$ Aleksandra E Zgierska ${ }^{3}$
}

To cite: Tuan W-J, Sehgal N, Zgierska AE. Using electronic health record's data to assess daily dose of opioids prescribed for outpatients with chronic non-cancer pain. Fam Med Com Health 2021;9:e001277. doi:10.1136/fmch-2021-001277

Check for updates

(C) Author(s) (or their employer(s)) 2021. Re-use permitted under CC BY-NC. No commercial re-use. See rights and permissions. Published by BMJ.

${ }^{1}$ Department of Family and Community Medicine, Penn State College of Medicine, Hershey, Pennsylvania, USA ${ }^{2}$ Department of Orthopedics \& Rehabilitation, University of Wisconsin-Madison School of Medicine and Public Health, Madison, Wisconsin, USA ${ }^{3}$ Department of Family and Community Medicine, Penn State Health Milton S Hershey Medical Center, Hershey,

Pennsylvania, USA

Correspondence to Dr Wen-Jan Tuan; wtuan@pennstatehealth.psu. edu

\section{ABSTRACT}

This research intended to examine electronic health record (EHR) based methods for automated estimation of morphine equivalent daily dose (MEDD) of prescribed opioids in primary care research and practice. The study leveraged the health system's audit of adults treated with long-term opioids for chronic non-cancer pain to compare two EHR-based automated MEDD calculation methods: RxSignature (active prescriptions' signature information) and RxQuantity (quantity dispensed for prescriptions issued within the past 90 days). Prescribed opioid EHR data were extracted from the target population at a large US academic health system in a 2-year assessment period. Forty-five 'target patients' were selected by the health system for a manual audit by an expert physician who then 'manually' calculated the actual MEDD over the past 90 days (RxAudit) for those with discrepancies in the MEDD calculated with RxSignature and RxQuantity. Paired samples t-test compared the MEDD generated by the RxSignature and RxQuantity methods by opioid type in the target population. The audit $(n=45)$ revealed the RxSignature and RxQuantity methods yielded comparable MEDD results for 20 patients and discrepant results for 25 patients. The former group had opioid prescriptions issued at regular intervals for stable, scheduled doses of opioids; the latter group had opioid prescriptions issued irregularly or for changed daily dosing regimen, for as-needed use, or had changes in the dosing regimen or inactive prescriptions mislabeled as active. RxAudit of the EHR of those with discrepant MEDD results $(n=25)$ produced consistent results with those yielded by the RxQuantity, but not the RxSignature, method. Significant differences in MEDD were found for most opioid types when the MEDD was calculated for the target population using the RxSignature and RxQuantity methods. In conclusion, different EHR-based methods for MEDD calculation can lead to vastly different estimates, with implications for research and clinical care outcomes. Standardising data extraction and MEDD calculation algorithms could overcome these challenges, enabling a more accurate and reproducible approach to the dose calculation for prescribed opioids, improving the quality of research and patient safety.

\section{INTRODUCTION}

Chronic non-cancer pain is one of the leading causes of disability and diminished quality of life in the USA, affecting over 50 million Americans and costing nearly $\$ 560$ billion annually in direct medical expenses, lost productivity and disability programmes. ${ }^{1}$ Many patients with chronic pain rely on long-term opioid therapy that can result in addiction, overdose and mental health deterioration while insufficiently addressing pain. ${ }^{2} 3$ While researchers and clinicians continue to improve interventions for safer pain care and opioid management, the effort to better understand and standardise analytical mechanisms for determining the dose of prescription opioids using the electronic health record (EHR) data have been limited. ${ }^{4}$ Yet, such efforts are urgently needed to support high-quality research and day-to-day clinical operations in primary care settings.

Advances in health information technology have enabled clinicians and health systems to quickly look up ongoing opioid prescriptions in the EHR platform for individual patients. Analytical functions for automatically estimating opioid dosage using the EHR data have been increasingly used to help better manage opioid dosage prescribed over time. ${ }^{56}$ It is important for clinicians to understand the system-wide computation processes for opioid-related metrics to ensure that care outcomes can be assessed in an informative and reliable way, in order to analyse opioid prescribing patterns, track dosage changes and advocate safer opioid prescribing practices. Having standardised and reliable EHRbased computation methodologies for automated calculation of morphine equivalent daily dose (MEDD) of prescribed 
opioids will further help researchers effectively evaluate clinically relevant outcomes and help inform care quality and patient safety among opioid-treated patients.

Despite availability of medication ordering information in EHR systems, reliable determination of morphine dose of prescribed opioids using the EHR data has remained challenging, due to variability in how the type and dose of the prescription opioid are converted and calculated in computational algorithms across analytical applications. ${ }^{78}$ Some methods may be estimating the daily opioid dose based on the current moment or active prescriptions; some may be accounting for all active prescriptions or prescriptions issued during a certain timeframe, while others may be calculating and reporting the dose per each prescription separately; some base their calculations on the prescription signature information, while others on the total number of prescribed units of a given opioid per prescription. Differences in the used conversion ratios for individual opioid medications and their preparations or route of administration additionally compound these problems. The inconsistency and ambiguity in MEDD measurement create complexity and barriers, which can negatively impact research, patient care and opioid therapy monitoring and management. For example, some computation algorithms could lead to unintentional overinflation of a given patient's MEDD, leading to erroneous research findings or inappropriate tapering decisions in clinical practices. ${ }^{9}$ Therefore, there is an urgent need for establishing a standardised methodology to consistently and accurately evaluate the prescription opioid dosage using the EHR data. The objectives of this study were to examine challenges related to the outcomes of current MEDD estimation methods, laying the foundation of the standardised approach for reliable MEDD estimation of prescribed opioids using the EHR data for research and clinical practice purposes.

\section{METHODS}

\section{Study design and setting}

This study was part of the health system's ongoing quality improvement (QI) effort focused monitoring and enhancing clinicians' adherence to the health system's opioid prescribing policy for chronic non-cancer pain. The methods for automated opioid data extraction and MEDD calculation were based on the EHR systemgenerated data and informed by the study team's prior and ongoing research projects, ${ }^{61011}$ which were approved by the Institutional Review Board. These two different methods were then applied as a part of the health system's quality of care audit to retrospectively examine their accuracy and consistency for automated MEDD calculation. The RxSignature method applied by the existing MEDD calculation algorithm using the signature information of prescriptions listed as 'active'. ${ }^{10}$ A prescription becomes inactive when it expires based on its end date (entered by the prescriber at the time of issuing this prescription) or when the clinician or clinical staff changes it to an inactive or complete status; if a prescription has no specified end date or is not deliberately changed to 'inactive', it can stay on the active medication list even if it not actually active. The RxQuantity method, developed by the study team, ${ }^{611}$ calculated MEDD based on the total quantity of the prescribed opioids over a specific period of time (past 90 days), regardless of the prescription status (active vs inactive) and without using the signature information. The results of these two automated EHR-based MEDD calculation methods were then compared in selected patients against the physician-conducted manual audit of the patient record (RxAudit). The manual audit is labour intensive and, as such, unfeasible for large-scale, system-wide ongoing QI monitoring efforts. Yet, it constitutes a gold standard in clinical practice for assessing the prescribing and other clinical practices when a given patient's care is evaluated, and, therefore, served as a reference for establishing the accuracy of the RxSignature and the RxQuantity MEDD calculation methods.

\section{Study population}

The study population included primary care patients of a large US academic centre who were at least 18 years old and prescribed long-term opioids (for at least 90 consecutive days) on the outpatient basis between 1 January 2018 and 31 December 2019; although the patients needed to have a primary care established at the health system, the outpatient opioids could have been prescribed by health system's primary care or specialty providers. The description of eligible opioids is presented below. Patients with the presence of cancer diagnoses (except non-melanoma skin cancer) or palliative/hospice care status in their problem list were excluded from the analysis. Data were extracted from an enterprise EHR database (Epic System, Verona, Wisconsin, USA).

\section{Eligible outpatient opioid prescriptions}

A variety of different pharmaceutical classes of opioids and numerous monotherapy and combination opioid medications can be prescribed to treat pain. To determine which opioid medications are used for treating chronic non-cancer pain in outpatient care, the study included 'opioid agonists' and 'opioid partial agonists', prescribed as monotherapy or as an 'opioid combination' medications that could be delivered in a form compatible with outpatient prescribing for chronic non-cancer pain (eg, pill, capsule, liquid, transdermal or transmucosal administration). Opioid medications/preparations determined by the reviewing study experts as administrated only in the inpatient, emergency department or other monitored clinical settings (eg, powder, intramuscular, intravenous or epidural administration preparations) or for pain indications other than chronic non-cancer pain (eg, palliative or cancer care) were excluded.

Methadone not prescribed by the health system clinicians and buprenorphine were not considered to be eligible opioids and were excluded from the analysis. 
Although methadone and buprenorphine can be used to treat chronic pain, they are also used to treat opioid use disorder (OUD). Both medications can be dispensed in the opioid treatment programme (OTP) settings to treat OUD. Both can be prescribed on the outpatient basis, outside of the OTP, to treat chronic pain. However, only buprenorphine can be prescribed in the regular clinics. At the time of this study, buprenorphine was primarily prescribed in the assessed health system for OUD care (typically prescribed in the sublingual form) but not for chronic pain (typically prescribed as the transdermal or buccal preparations) and was, therefore, considered an ineligible opioid. Methadone was considered as eligible and included in the analysis when it was prescribed by the health system clinicians, as such prescriptions were only possible for chronic pain indications. Methadone prescriptions were excluded when they were noted as a historical medication because such documentation suggested this medication was issued outside of the assessed health system, most likely to treat OUD within the OTP settings.

A review of the enterprise EHR database revealed 22 pharmacologically unique opioids, with 1824 corresponding distinct opioid-containing medications prescribed in the outpatient settings. Their names, dose per unit and route of delivery information were extracted. All distinct medications were reviewed by the study team to determine their eligibility to verify the unit dose of an opioid component in combination medications.

\section{Opioid dose calculation and validation}

Literature in chronic pain and addiction medicine fields recommends the use of MEDD as one of the measures to assess the risk/benefit of various opioid dosage levels. ${ }^{12}$ The MEDD represents a patient's daily opioid dose, expressed in morphine milligram equivalents (MMEs). The MEDD is calculated by multiplying the total daily dose of a given opioid by the established conversion factor to arrive at this opioid's daily MME, then summing the daily MME for all different opioids taken by the patient. For this study, each unique opioid was assigned a conversion factor specific to this opioid and its route of administration (table 1) following the recommended conversion factors, ${ }^{13} 14$ which were crossexamined by the study's expert physicians (AEZ and NS).

Long-term opioid therapy is defined as lasting for at least 90 days. ${ }^{15} 16$ With the focus of this study on patients treated with long-term opioid therapy, we established the 'prior 90 days' as the most appropriate assessment period when calculating the MEDD using the RxQuantity and RxAudit methods. Relying on a longer assessment period (eg, prior 90 days), rather than on the current prescriptions only, for assessing the MEDD is consistent with clinical practice guidelines, ${ }^{17}$ which recommend evaluating the patient's adherence to opioid therapy by checking their prior prescription records in the EHR and Prescription Drug Monitoring
Table 1 Morphine milligram equivalent conversion factors for the 10 opioid types prescribed to the target population $^{1314}$

\begin{tabular}{lll}
\hline Opioid & Unit & Factor \\
\hline Codeine & $\mathrm{mg}$ & 0.15 \\
\hline Fentanyl transdermal patch & $\mu \mathrm{g} / \mathrm{hour}$ & 2.4 \\
\hline Hydrocodone & $\mathrm{mg}$ & 1 \\
\hline Hydromorphone & $\mathrm{mg}$ & 4 \\
\hline Methadone & & \\
\hline$(1-20)$ & $\mathrm{mg}$ & 4 \\
\hline$(21-40)$ & $\mathrm{mg}$ & 8 \\
\hline (41-60) & $\mathrm{mg}$ & 10 \\
\hline (>60) & $\mathrm{mg}$ & 12 \\
\hline Morphine & $\mathrm{mg}$ & 1 \\
\hline Oxycodone & $\mathrm{mg}$ & 1.5 \\
\hline Oxymorphone & $\mathrm{mg}$ & 3 \\
\hline Tapentadol & $\mathrm{mg}$ & 0.4 \\
\hline Tramadol & $\mathrm{mg}$ & 0.1 \\
\hline
\end{tabular}

databases for signs of early refills or 'doctor shopping'. Factoring such adherence findings into the decisionmaking process prior to issuing a new prescription is critical for reducing the risk of opioid misuse, overdose and diversion.

As part of the health system-wide ongoing QI effort, patients treated with long-term opioid therapy for chronic non-cancer pain were identified for quality monitoring and enhancement. From the sample, 45 patients were randomly selected, using a simple random sampling method, by the health system's QI auditing team for manual chart review. For these patients, the MEDDs calculated using the health system's RxSignature method and using the study-developed RxQuantity method were compared. Among these 45 patients, the MEDD values calculated using the two automated methods were consistent for 20 patients and discrepant for 25 patients. The medication detail field of the audit data, listing all opioid prescriptions for the past 90 days, was reviewed for these 45 patients. In addition, a 'manual' in-depth EHR review (RxAudit) was completed (AEZ) for patients with discrepant MEDD values produced by the RxSignature and RxQuantity methods as the next step of the health system-initiated audit, enabling the accuracy assessment of the two automated MEDD calculation methods against the findings of expert-conducted audit.

Therefore, the MEDD assessment approaches included:

(A) RxSignature method: the prescription signaturebased MEDD was available to clinicians through the health system's EHR (Epic Clarity database, version 2018).$^{10}$ It was generated using the signature field of each opioid prescription listed as the 'active medication'. The signature field contains instructions for patients on how to use the medication, that is, the 
medication dose and frequency. Following the RxSignature method approach, the EHR system calculates the MEDD for each opioid prescription listed as the active medication by parsing the name, strength and dosage information stored in the signature field. For multiple active opioid prescriptions, the RxSignature method calculates MEDD as the sum of MEDD of all opioid prescriptions listed as 'active' at the time of calculation; this method does not account for prescriptions outside of the 'active medication' list. The RxSignature method, by focusing on the signature field, needs at times to make assumptions about the daily medication use; for example, a prescription for $5 \mathrm{mg}$ hydrocodone ('take $5 \mathrm{mg}$ every 4 hours as needed', dispense five tablets) would calculate the MEED value as $30 \mathrm{mg}(5 \mathrm{mg} \times$ up to six times/day), disregarding the number of total doses prescribed (five dispensed tablets of $5 \mathrm{mg}$ hydrocodone could yield a maximum dose of $25 \mathrm{mg}$ if all taken within 1 day), and when the prescription was issued, as long as it was still labelled as active. RxSignature therefore calculated MEDD in the following way:

MEDD $=\sum$ daily MME (strength $/$ unit $\times$ presumed quantity $/$ day $\times$ MME conversion factor)

of each opioid prescription listed as active at the time of calculation

(B) RxQuantity method: this method was developed by the study team, ${ }^{611}$ based on the previous work,${ }^{15} 16$ to estimate the average MEDD prescribed to each patient within a 90-day assessment period. The RxQuantity method computes the MEDD based on all opioid prescriptions issued within the prior 90-day window, regardless whether the prescription is labelled as 'active' or not; a prescription could have become inactive due to its end date having passed or the clinical staff marking it as such; however, if it was issued within the assessment window, it could have been filled and its 'dispensed quantity' counted towards the MEDD calculation. Under the RxQuantity method, for each opioid prescription issued within the past 90 days, each opioid's unit dose is multiplied by its MME conversion factor and by its total prescribed quantity (the 'dispense' field of each prescription) to yield each opioid prescription's MEDD. Then, the MEDDs of all identified opioid prescriptions are added, before dividing this sum by 90 to calculate the average daily opioid dose over the past 90 days. The formula for calculating MEDD for the RxQuantity method is as follows:

MEDD $=\sum$ total MED per each prescription (strength $/$ unit $\times$ total

$\times$ MME conversion factor) issued within the past 90 days/90

(C) RxAudit method: a physician (AEZ) experienced in the EHR data extraction, pain management standards and opioid prescribing guidelines manually reviewed and extracted data related to opioid prescribing from the EHR record for patients selected for the RxAudit. Each chart review assessed the EHR data for opioid prescriptions issued within the past 90 days and, if additional information was needed, from clinical notes (eg, clinic visit or phone/online message free-text fields) to accurately determine the prescribed MEDD over the past 90 days.

\section{Comparison of MEDD between the two automated EHR-based computation methods}

To compare the difference in MEDD calculated by the RxSignature and the RxQuantity methods, the MEDD difference yielded by these two methods was calculated for the identified 10 opioid types prescribed in the outpatient settings in the study population $(n=3022)$. More specifically, the MEDD difference was first computed by subtracting the MEDD calculated by the RxQuantity method from that calculated by the RxSignature method and presented as mean value (SD and 95\% CIs) The onesample Kolmogorov-Smirnov normality test showed $\mathrm{p}$ values equal to or greater than 0.05 for all opioid types, indicating that MEDD differences were normally distributed. A t-test for independent samples was applied to compare differences in MEDD calculated using two automated methods. The statistical significance level was set at a two-tailed $\mathrm{p}<0.05$. The statistical analyses were performed using SAS V.9.3 software (SAS Institute Inc).

\section{RESULTS}

\section{Study population characteristics}

The target population consisted of 3022 primary care adult patients treated for chronic non-cancer pain with long-term opioids between 1 January 2018 and 31 December 2019 (figure 1). During this 2-year period, 52634 outpatient opioid prescriptions were ordered for this population, including 10 unique opioid types: oxycodone $(40.7 \%)$, hydrocodone $(29.0 \%)$, morphine $(12.5 \%)$, tramadol $(8.3 \%)$, fentanyl $(3.7 \%)$, methadone $(3.0 \%)$, codeine $(1.5 \%)$, hydromorphone $(1.1 \%)$ and

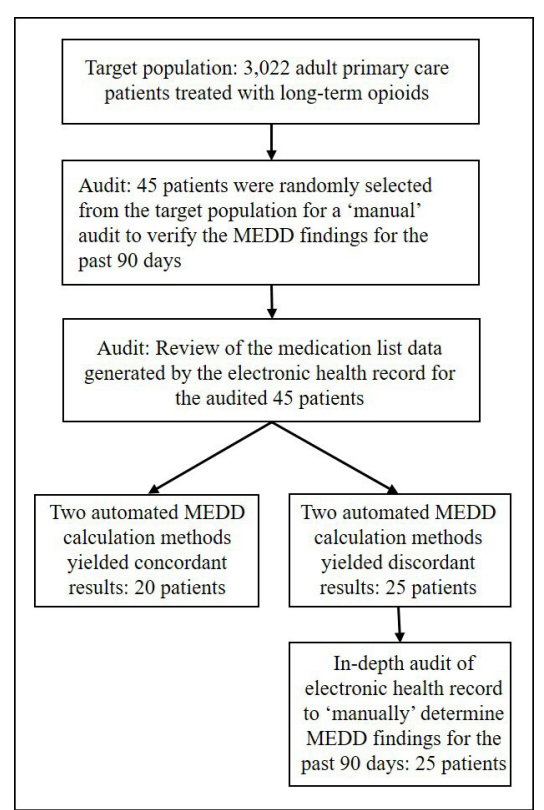

Figure 1 Flow chart of the opioid type and patient sample selection process. MEDD, morphine equivalent daily dose 
oxymorphone $(0.1 \%)$. The top five most frequently prescribed opioids accounted for more than $94.3 \%$ of all opioid prescriptions issued during the assessment period.

\section{MEDD results yielded by the three computational methods}

Forty-five individuals were randomly selected from the target population as a part of the health system's routine quality of care audit. Using their EHR data, the MEDD calculated from the RxSignature and RxQuantity methods produced similar results for 20 and discrepant results for 25 of these audited patients. Audit-extracted data on prescribed opioids indicated that 20 patients with concordant RxSignature and RxQuantity MEDD results were prescribed with a stable daily dose of scheduled opioids, prescribed 'when due' during the assessed 90-day period.

Manual review of EHRs of each of the 25 patients with discrepancies in the RxSignature and RxQuantity methods produced MEDD values (RxAudit) showed that, in this group, opioid prescriptions changed, were issued irregularly, for an as-needed use, or were outside of the assessment period yet (inaccurately) still labelled as active. For the 25 manually audited patients, the mean RxSignature-based MEDD was greater than the mean RxQuantity-based MEDD (140.3 $\pm 152.9 \mathrm{MME} /$ day vs $102.3 \pm 139.2 \mathrm{MME} /$ day, respectively; pairwise t-test: $\mathrm{t}=2.83, \mathrm{p}<0.01)$. The RxAudit-based MEDD values were consistent with the RxQuantity-based estimates.

The examples below illustrate the scenarios when the RxSignature and RxQuantity yielded discrepant MEDD values, and how these results compared with the RxAuditbased MEDD findings. The MEDD calculations for each case and each method are detailed in table 2.

\section{Case 1}

The medication list showed one opioid prescription issued in the past 90 days, labelled as 'active', and issued for $4 \mathrm{mg}$ hydromorphone tablets to take as-needed every 6 hours. Using the RxSignature method, the MEDD was estimated at 64 morphine mg equivalents (MME)/day. This estimate was higher than the MEDD yielded by the RxQuantity and RxAudit methods, which both resulted in 24.9 MME/day on average over the past 90 days.

\section{Case 2}

The EHR medication list showed three consecutive prescriptions, all issued in the past 90 days, for $4 \mathrm{mg}$ oxycodone tablets to take two tablets every 4-6 hours as needed; the end dates were specified for each prescription, and only the most recent prescription was marked as 'active'. The RxSignature method, using the signature information derived from the one active prescription, computed the MEDD as $30 \mathrm{MME} /$ day. The MEDD estimated by both the RxQuantity and RxAudit methods was 32.7 MME/day on average over the past 90 days.

\section{Case 3}

The medication list showed two prescriptions issued within the past 90 days and labelled as 'active': one for morphine extended release $30 \mathrm{mg}$ tablets (take one twice a day) and another for hydrocodone $4 \mathrm{mg}$ tablets (take one every 4 hours as needed). The RxSignature method estimated MEDD at $90 \mathrm{MME} /$ day; both the RxQuantity and the RxAudit methods resulted in the MEDD of 23.3 $\mathrm{MME} /$ day on average over the past 90 days.

\section{Case 4}

The medication list showed two 'active' opioid prescriptions: one for $100 \mu \mathrm{g} /$ hour fentanyl patch (apply one every 3 days) and another for $5 \mathrm{mg}$ oxycodone tablets (take 2 every 4 hours as needed). Although they were both issued 6 months prior to the audit date, they did not have an 'end date' entered and were not marked as 'completed'; therefore, they were still labelled as 'active' in the EHR, and the RxSignature yielded MEDD of 330 MME/day based on these two prescriptions. Because these prescriptions were issued outside of the 90-day assessment period, the RxQuantity-based and RxAuditbased MEDD was zero.

\section{Comparing MEDD between the two automated EHR-based computation methods}

During the study assessment period, the 3022 target patients were issued a total of 52644 prescriptions for outpatient opioid medications, derived from 10 opioid types. The difference in MEDD calculated using the RxSignature and RxQuantity methods was examined by each opioid type (table 3 ). The two methods produced different MEDD estimates $(\mathrm{p}<0.05)$ for fentanyl, oxymorphone, codeine, hydromorphone, hydrocodone, meperidine, oxycodone, tramadol and morphine, with the first four opioids noted to have the largest MEDD discrepancies. Differences in MEDD between the RxSignature and the RxQuantity methods were not statistically significant for tapentadol $(\mathrm{p}=0.49)$.

\section{DISCUSSION}

MME daily dose of opioids has been ubiquitously used in research settings and considered a standard metric when assessing the benefit and risk of opioids prescribed for pain management in routine clinical practice. MEDD is used by clinicians and researchers to inform therapeutic strategy and serve as a risk predictor for potential opioidrelated harm, particularly overdose. The MEDD can be accurately completed through the 'manual' review of individual patient EHRs and prescription records; yet, this approach is time consuming and labour intensive and requires specialised skills to estimate the average opioid dose over a specific time period. The manual MEDD calculation process is also error prone. As such, the manual review of individual patient EHR is subpar and unfeasible for research or clinical purposes involving numerous, regular audits and large patient populations.

To overcome the shortcomings of a manual audit, modern EHR systems have adopted ways for automating the MEDD calculation as part of the EHR-based clinical 
Table 2 Case examples illustrating the different MEDD computation methods and the MEDD results yielded by each method

\section{Case description}

Case 1:

Prescription 1 (active; issued within the past 90 days): hydromorphone $4 \mathrm{mg}$ tablets, take $0.5-1$ tablets every 6 hours as needed. Quantity: 140 tablets.

\section{Case 2:}

Prescription 1 (active; issued within the past 90 days): oxycodone $5 \mathrm{mg}$ tablets, take 1-2 tablets every 4 hours as needed. Quantity: 140 tablets.

Prescription 2 (inactive; issued within the past 90 days): oxycodone $5 \mathrm{mg}$ tablets, take 1-2 tablets 3-4 times daily as needed, maximum four tablets daily. Quantity: 140 tablets.

Prescription 3 (inactive; issued within the past 90 days): oxycodone $5 \mathrm{mg}$ tablets, take 1-2 tablets twice a day as needed. Quantity: 112 tablets.

\section{Case 3:}

Prescription 1 (active; issued within the past 90 days): morphine extended release 30 mg tablets, take 1 tablet twice a day. Quantity: 60 tablets. Prescription 2 (active; issued within the past 90 days): hydrocodone $5 \mathrm{mg}$ tablets, take 1 tablet every 4 hours as needed. Quantity: 60 tablets.

\section{Case 4:}

Prescription 1 (active; issued prior to the past 90 days): fentanyl patch $100 \mu \mathrm{g} / \mathrm{hour}$, apply one patch to skin every 3 days.

Quantity: 10 patches.

Prescription 2 (active; issued prior to the past 90 days): oxycodone 5 mg tablets, take 1-2 tablets every 4 hours as needed. Quantity: 270 tablets.

\section{MEDD computation using different methods}

MEDD

RxSignature: the signature information allows to take up to 1 tablet four times per day. $\quad 64$ The MEDD calculated based on these instructions:

$4 \mathrm{mg}$ hydromorphone/tablet $\times 4$ conversion factor $\times 4$ times/day dosing=64 MME/day.

RxQuantity: a total of 140 hydromorphone tablets were prescribed during a 90-day

period. MEDD computed based on this quantity during a 90-day period:

( $4 \mathrm{mg}$ hydromorphone/tablet $\times 4$ conversion factor $\times 140$ tablets) $/ 90$ days $=24.9 \mathrm{MME} /$ day.

RxAudit: the manual audit confirmed the presence of a single prescription during the 90-day assessment window. MEDD computed based on this quantity during a 90-day period:

( $4 \mathrm{mg}$ hydromorphone/tablet $\times 4$ conversion factor $\times 140$ tablets) $/ 90$ days $=24.9$ morphine equivalent $\mathrm{mg} /$ day.

RxSignature: the MEDD was calculated based on prescription 1 (the only active prescription), using its signature field: oxycodone $5 \mathrm{mg} /$ tablet $\times 1.5$ conversion factor $\times 2$ tablets/dose $\times 4$ times/day=60 MME/day.

RxQuantity: the total quantity of oxycodone prescribed during the 90-day window was used to compute the MEDD: (oxycodone $5 \mathrm{mg} /$ tablet $\times 1.5$ conversion factor $\times(140+140+112$ tablets $)) / 90$ days $=32.7 \mathrm{MME} /$ day .

RxAudit: the auditing team manually reviewed progress notes and prescription data. The patient was given a total of 292 tablets of $5 \mathrm{mg}$ oxycodone within 90 days. The calculated MEDD during a 90-day period was:

(oxycodone $5 \mathrm{mg} /$ tablet $\times 1.5$ conversion factor $\times(140+140+112$ tablets) $) / 90$ days $=32.7$ MME/day.

RxSignature: both prescriptions were active at the time of calculation. The MEDD calculated based on their signature instructions: (morphine $30 \mathrm{mg} /$ tablet $\times 1$ conversion factor $\times 2$ tablets/day)+(hydrocodone $5 \mathrm{mg} /$ tablet $\times 1$ conversion factor $\times 6$ times/day) $=90 \mathrm{MME} /$ day .

RxQuantity: the MEDD calculation was based on the quantity of prescribed

medications over the past 90 days:

((morphine $30 \mathrm{mg} /$ tablet $\times 1$ conversion factor $\times 60$ tablets)+(hydrocodone $5 \mathrm{mg} /$ tablet $\times 1$ conversion factor $\times 60$ tablets $) / 90$ days $=23.3 \mathrm{MME} /$ day .

RxAudit: the manual audit confirmed a stable dose of morphine of $60 \mathrm{mg} / \mathrm{day}$ and a single prescription for as-needed hydrocodone during the assessment period. The calculated MEDD during a 90-day period was:

((morphine $30 \mathrm{mg} /$ tablet $\times 1$ conversion factor $\times 60$ tablets)+(hydrocodone $5 \mathrm{mg} /$ tablet $\times 1$ conversion factor $\times 60$ tablets) $) / 90$ days $=23.3 \mathrm{MME} /$ day.

RxSignature: at the time of calculation, prescriptions 1 and 2 were labelled as active.

The MEDD calculated based on these instructions of the two prescriptions:
(fentanyl patch $100 \mu \mathrm{g} /$ hour $\times 2.4$ conversion factor $\times 1$ patch) + (oxycodone $5 \mathrm{mg} /$ tab $\times 1.5$ conversion factor $\times 2$ tabs $\times 6$ times/day) $=330 \mathrm{MME} /$ day .

RxQuantity: because the prescriptions were issued outside of the assessment period (more than 90 days prior to the calculation date), the MEDD during the past 90 days was zero.

RxAudit: because the prescriptions were issued outside of the assessment period (more than 90 days prior to the calculation date), the MEDD during the past 90 days was zero.

MEDD, morphin equivalent daily dose.

decision support tools. ${ }^{10} 11$ Although these approaches seem straightforward, the actual calculation process is complex and non-standardised across different EHR systems; even the individual prescription details are not always presented in a standardised structure or a codified format. This study found notable discrepancies in MEDD when apply different automated calculation methods. The discrepancies were more likely to be present in cases 
Table 3 Comparison in MEDD between the RxSignature method and the RxQuantity method, for opioids prescribed in the 2-year study period

\begin{tabular}{|c|c|c|c|c|c|}
\hline Opioid & Prescriptions, total number & MEDD difference ${ }^{\star}$, mean (SD) & $95 \% \mathrm{Cl}$ & t-statistic & $P$ value \\
\hline Codeine & 476 & $23.9(2.8)$ & 18.3 to 29.4 & 8.4 & $<0.01$ \\
\hline Fentanyl & 2382 & $-80.1(5.9)$ & -91.8 to -68.4 & -13.4 & $<0.01$ \\
\hline Hydrocodone & 16885 & $16.6(1.2)$ & 14.2 to 19.0 & 13.5 & $<0.01$ \\
\hline Hydromorphone & 551 & $21.6(1.8)$ & 17.9 to 25.2 & 11.7 & $<0.01$ \\
\hline Methadone & 18 & $12.6(0.9)$ & 10.9 to 14.3 & 15.5 & $<0.01$ \\
\hline Morphine & 7600 & $5.5(1.0)$ & 3.6 to 7.4 & 5.7 & $<0.01$ \\
\hline Oxycodone & 21949 & $10.4(0.3)$ & 9.8 to 11.0 & 34.7 & $<0.01$ \\
\hline Oxymorphone & 105 & $-29.9(8.4)$ & -46.4 to 13.4 & -3.6 & $<0.01$ \\
\hline Tapentadol & 67 & $-2.0(2.9)$ & -7.7 to 3.7 & -0.7 & 0.49 \\
\hline Tramadol & 2598 & $5.8(0.2)$ & 5.4 to 6.2 & 27.5 & $<0.01$ \\
\hline
\end{tabular}

*MEDD difference was calculated as the subtraction of RxQuantity MEDD from RxSignature MEDD (ie, positive difference value indicates RxSignature MEDD was greater than RxQuantity MEDD).

MEDD, morphine equivalent daily dose.

where prescriptions were issued 'too early' (ie, before the patient's prior prescription period expired), changes were made to the opioid medications dose or use frequency during the assessment period or-the most common case-when opioids were prescribed for 'as needed' use. Specifically, the RxSignature method only accounts for prescriptions labelled as 'active' at the time of MEDD calculation. As the active medications lists change over time, RxSignature MEDD may vary during the assessment period. Moreover, the literature indicates that current EHR systems often apply prespecified rule-based algorithms to parse free-text signature information to identify the type, strength, timing and frequency of prescribed opioids. ${ }^{10}$ Although the signature field in EHRs may be constructed in a machine-interpretable presentation, potential spelling errors, ambiguous descriptions or non-standard instructions may lead to misinterpretation or missing outputs. The computation process can also become complicated when multiple prescriptions in different timeframes are involved.

The study findings raise concerns about potential imprecision and ambiguity in using the signature-based method to determine the dose and frequency of the opioid prescription. Signature instructions often have less specific frequency and dosage recommendations (ie, take two to three tablets four to six times a day as needed). The implicit instructions require computation algorithms to make inferences or assumptions about the timing and dose of medication administration. The ambiguous translation nature of the algorithm can increase the risk of underestimating or overestimating the actual MEDD. It also can lead to potential misclassification of overall opioid dosage, particularly for patients with multiple prescribed opioids. This may unintentionally lead to inaccurate results and conclusions in research, and misguided clinical decisions, such as forced or accelerated tapering in those identified as treated with high MEDD. ${ }^{9}$
Overall, no discrepancies were noted between the MEDD calculated with the RxSignature and RxQuantity methods in patients treated with a stable dose of scheduled opioids during the assessment period, with prescriptions issued regularly, at the time when they were due. This was not the case for patients with irregularly issued, changing or imprecise (eg, as-needed dosing) prescriptions. Although the RxSignature and RxQuantity are expected to yield different results because they are based on different computational algorithms, the RxQuantity conceptual approach is consistent with clinical practice, which encourages clinicians to account for prior/other opioid prescriptions (eg, by checking the EHR for the past prescriptions or checking records in the Prescription Drug Monitoring Program) when determining the risk of the current therapy and prior to considering a new opioid prescription. This MEDD computational strategy based on the 'dispense quantity' for all prescriptions issued within a certain timeframe (eg, 90 days to be aligned with the definition of long-term opioid therapy), rather than the signature instructions of active prescriptions, uses discrete fields in the medication order records to lessen the risk of interpretation errors resulting from the imprecision of the signature-based information. Our study indicated the RxQuantity and the RxAudit methods produced consistent MEDD estimates. The RxQuantity approach has been used in prior research examining clinician adherence to opioid prescribing guidelines and its impact on patient outcomes in primary care settings. ${ }^{6} 11^{18}$ Overall, the RxQuantity, if implemented across the research, clinical and public health settings, could standardise the MEDD calculation, enabling a unified approach to assessing the benefit and risk of and response to prescription opioids. The use of the actual dispense quantity and dates can further help clinicians ascertain the overall amount of opioid dose prescribed to patients over a specific period and could provide greater 
consistency in monitoring prescribing opioids in EHRs to improve care quality and patient safety at primary care practice.

Our study highlights potential challenges that exist different MEDD computation algorithms. As researchers continue to enhance EHR-based algorithms for estimating MEDD, the quantity-based computation method can serve as a complement to the signature-based method for future research and clinical practices. The MEDD computation methods examined in this study present a conceptual approach aimed at standardising MEDD calculation using the EHR data and could be adapted and applied to any EHR platform or other large databasesboth in and outside the USA-containing data on opioid prescriptions.

\section{Limitations}

Our findings are based on EHR-based ambulatory prescription records, with manual audit completed for a small, yet randomly generated, group of patients. The results may not be generalisable to other settings, such as when opioids are directly administered for the emergency department or inpatient care. A small sample of patient records audited to manually verify the accuracy of automated MEDD estimates, limits generalisability of our results, calling for further, larger studies. In addition, the MEDD estimation in this study was based on medication order records; this system does not note whether patients actually filled the prescription, took their medications at home as prescribed (or at all) or obtained opioids from other sources (eg, additional prescriptions were issued outside of the assessed health system); these limitations, although inherent to the EHR data, may contribute to either underestimation or overestimation of the actual MEDD taken by individual patients. Therefore, augmenting the EHR-based data on prescribed opioids, with the claims data on insurance-based dispensed opioid prescriptions, the prescription drug monitoring program (PDMP)-based data on dispensed opioids and with clinical information (eg, patient report and urine toxicology testing results) are necessary for optimal understanding of research findings an clinical decision making.

\section{CONCLUSION}

Understanding the dosage estimation mechanisms can be important for researchers and clinicians to ensure that research findings and care outcomes can be evaluated in a systematic and system-wide fashion. The EHR-based automated calculation of the daily dose of prescription opioids, using the method accounting for the quantity of opioids dispensed over a certain period of time (eg, past 90 days), could provide a practical and reproducible measure of MEDD that could be implemented in the research, clinical and public health settings, creating a foundation for standardised assessment of reporting of MEDD.
Contributors All authors were involved in revisions, read and approved the final manuscript. W-JT extracted electronic health record data, performed statistical analysis, contributed to writing the manuscript, and responsible for overall content as the guarantor. NS reviewed morphine milligram equivalent convention factors, provided guidance on the results and contributed to writing the manuscript. AEZ reviewed morphine milligram equivalent convention factors, performed chart review for comparing MEDDs among different computation methods and contributed to writing the manuscript.

Funding The project was funded through a Patient-Centered Outcomes Research Institute (PCORI) Award (OPD-1601-33860). This project also received funding and institutional support through University of Wisconsin-Madison School of Medicine and Public Health, and its Department of Family Medicine and Community Health; Brigham and Women's Hospital, Harvard Medical School; University of Utah College of Social Work; and Pennsylvania State University Department of Family and Community Medicine.

Disclaimer The views and statements in this publication are solely the responsibility of the authors and do not necessarily represent the views of the PCORI, its Board of Governors or Methodology Committee.

Competing interests None declared.

Patient consent for publication Not applicable.

Ethics approval The research was approved by the university's institutional review board (ID: 2017-0353).

Provenance and peer review Not commissioned; externally peer reviewed.

Data availability statement № data are available.

Open access This is an open access article distributed in accordance with the Creative Commons Attribution Non Commercial (CC BY-NC 4.0) license, which permits others to distribute, remix, adapt, build upon this work non-commercially, and license their derivative works on different terms, provided the original work is properly cited, appropriate credit is given, any changes made indicated, and the use is non-commercial. See: http://creativecommons.org/licenses/by-nc/4.0/.

\section{ORCID iD}

Wen-Jan Tuan http://orcid.org/0000-0003-3939-8979

\section{REFERENCES}

1 Dahlhamer J, Lucas J, Zelaya C, et al. Prevalence of Chronic Pain and High-Impact Chronic Pain Among Adults - United States, 2016. MMWR Morb Mortal Wkly Rep 2018;67:1001-6.

2 Dunn KE, Barrett FS, Fingerhood M, et al. Opioid overdose history, risk behaviors, and knowledge in patients taking prescribed opioids for chronic pain. Pain Med 2017;18:1505-15.

3 Rogers AH, Bakhshaie J, Buckner JD, et al. Opioid and cannabis CoUse among adults with chronic pain: relations to substance misuse, mental health, and pain experience. J Addict Med 2019;13:287-94.

4 Liu H, Burkhart Q, Bell DS. Evaluation of the NCPDP structured and Codified SIG format for e-prescriptions. J Am Med Inform Assoc 2011;18:645-51.

5 Norman JL, Kroehl ME, Lam HM, et al. Implementation of a pharmacist-managed clinic for patients with chronic nonmalignant pain. Am J Health Syst Pharm 2017;74:1229-35.

6 Zgierska AE, Robinson JM, Lennon RP, et al. Increasing system-wide implementation of opioid prescribing guidelines in primary care: findings from a non-randomized stepped-wedge quality improvement project. BMC Fam Pract 2020;21:245.

7 Dasgupta N. Inches, centimeters, and yards: overlooked definition choices inhibit interpretation of morphine equivalence. Oral presentation at: U.S. Food \& Drug Administration (FDA) workshop for morphine milligram equivalents, 2021. Available: https://www. fda.gov/drugs/news-events-human-drugs/morphine-milligramequivalents-current-applications-and-knowledge-gaps-researchopportunities-and [Accessed 19 Aug 2021].

8 Rennick A, Atkinson T, Cimino NM, et al. Variability in opioid equivalence calculations. Pain Med 2016;17:892-8.

9 U.S. Food and Drug Administration (FDA). FDA identifies harm reported from sudden discontinuation of opioid pain medicines and requires label changes to guide prescribers on gradual, individualized tapering, 2019. Available: https://www.fda.gov/drugs/ drug-safety-and-availability/fda-identifies-harm-reported-suddendiscontinuation-opioid-pain-medicines-and-requires-label-changes [Accessed 20 Aug 2021]. 
10 Goud A, Kiefer E, Keller MS, et al. Calculating maximum morphine equivalent daily dose from prescription directions for use in the electronic health record: a case report. JAMIA Open 2019;2:296-300.

11 Quanbeck A, Brown RT, Zgierska AE, et al. A randomized matchedpairs study of feasibility, acceptability, and effectiveness of systems consultation: a novel implementation strategy for adopting clinical guidelines for opioid prescribing in primary care. Implement Sci 2018;13:21.

12 Liebschutz JM, Xuan Z, Shanahan CW, et al. Improving adherence to long-term opioid therapy guidelines to reduce opioid misuse in primary care: a cluster-randomized clinical trial. JAMA Intern Med 2017;177:1265-72.

13 Centers for Disease Control and Prevention. Analyzing prescription data and morphine milligram equivalents (MME). Available: https:// www.cdc.gov/drugoverdose/ resources/data.html[Accessed $10 \mathrm{Apr}$ 2018].
14 Centers for Medicare and Medicaid. Opioid oral morphine milligram equivalent (MME) conversions factors. Available: https://www.cms. gov/Medicare/Prescription-Drug-coverage/PrescriptionDrugCovC ontra/Downloads/Opioid-Morphine-EQ-Conversion-Factors-Aug2017.pdf [Accessed 21 Apr 2018].

15 Von Korff M, Korff MV, Saunders K, et al. De facto long-term opioid therapy for noncancer pain. Clin J Pain 2008;24:521-7.

16 Deyo RA, Smith DHM, Johnson ES, et al. Opioids for back pain patients: primary care prescribing patterns and use of services. J Am Board Fam Med 2011;24:717-27.

17 Dowell D, Haegerich TM, Chou R. CDC Guideline for Prescribing Opioids for Chronic Pain - United States, 2016. MMWR Recomm Rep 2016;65:1-49.

18 Zgierska AE, Vidaver RM, Smith P, et al. Enhancing system-wide implementation of opioid prescribing guidelines in primary care: protocol for a stepped-wedge quality improvement project. $B M C$ Health Serv Res 2018;18:415. 